\title{
Judgments of Learning in Context: Backgrounds Can Both Reduce and Produce Metamemory Illusions
}

\author{
Joshua R. Tatz ${ }^{1}$ - Zehra F. Peynircioğlu ${ }^{1}$ \\ Published online: 15 November 2019 \\ (C) The Psychonomic Society, Inc. 2019
}

\begin{abstract}
Varying item-specific features such as size (Rhodes \& Castel, 2008) or blur (Yue, Castel, \& Bjork, 2013) often produces metamemory illusions in which one type of item receives higher judgments of learning (JOLs) without being recalled better. In this study, we explored how similar manipulations to context would influence JOLs. When to-be-recalled words varying in size (or blur) were accompanied by backgrounds also varying in size (or blur), the traditional JOL illusions were reduced (Experiments 1, 2, 4, and 5) compared to when there were no backgrounds (Experiments 3a, 3b, and 4). Thus, the itemspecific and contextual cues were used interactively. Further, the background manipulations also sometimes themselves led to metamemory illusions regarding JOLs for the to-be-remembered items. In general, there were robust individual differences in how participants used the cues, including how they incorporated the contextual cues into their JOL decisions. In part, this may explain why interactive cue utilization did not always emerge at the group level. In sum, we showed that context may affect JOLs both directly and indirectly by influencing participants' use of item-specific cues. These findings broaden our understanding of how cues may be utilized (e.g., Koriat, 1997) and integrated (e.g., Undorf, Söllner, and Bröder, 2018) in JOLs.
\end{abstract}

Keywords JOL $\cdot$ Cue utilization $\cdot$ Cue integration $\cdot$ Font size $\cdot$ Individual differences

Judgments of learning (JOLs) are metacognitive assessments individuals make about how well something is learned. Experimentally, this usually takes the form of subjective likelihood ratings concerning future remembering. In general, participants' JOLs are predictive of memory performance (e.g., Arbuckle \& Cuddy, 1969); however, the relationship is not perfect. Often, factors that do not impact memory performance can nevertheless affect participants' JOL ratings. One, now classic, example of this is the font size effect, in which participants predict memory to be better for words presented in larger fonts during study despite a failure to recall such items better (Rhodes \& Castel, 2008). Factors, such as font size, on which participants base their JOLs are known as "cues." Currently, the most successful model in explaining how JOLs are based on cues is Cue-Utilization Theory

Joshua R. Tatz

Joshua.tatz@gmail.com

1 Department of Psychology, American University, Washington DC 20016 USA
(Koriat, 1997), which proposes that individuals base their JOLs on heuristics present at the time of learning.

Cue utilization refers to the degree to which individuals make use of various factors to predict their future memory performance, whereas cue validity refers to the degree to which those predictions actually align with performance (Koriat, 2008). In addition, Cue-Utilization Theory (Koriat, 1997) draws a distinction between intrinsic cues that pertain to features inherent to the study items (e.g., the semantic relatedness of paired associates) and extrinsic cues that pertain to features unrelated to the study items per se (e.g., item repetition). In general, participants can make accurate predictions about memory performance using both cue types (cue validity); however, extrinsic cues tend to influence JOLs less than intrinsic cues even when they are actually more influential to performance (cue utilization) (e.g., Zechmeister \& Shaughnessy, 1980; Dunlosky \& Nelson, 1994).

Extrinsic cues may, however, be utilized more when cue differences are made more apparent across trials (e.g., Dunlosky, Hunt, \& Clark, 2000). For instance, Castel (2008) found that participants' JOLs underestimated the memory benefits of primacy and recency during list learning, but that this result was ameliorated, and participants' JOLs tracked memory performance better, when the target item's position 
in the list (e.g., first) was explicitly stated in repeated studytest cycles. Thus, explicitly drawing participants' attention to extrinsic cues can enhance cue utilization.

With respect to physical attributes of items, font size is typically considered an intrinsic cue (e.g., Rhodes, 2016), which is consistent with Koriat's (1997) definition of intrinsic cues involving intra-item features. Undorf, Söllner, and Bröder (2018) suggest that one test for classifying cues as intrinsic or extrinsic might be whether the cue can be separated from the study item. By this logic, they consider font size to be ambiguous (i.e., a word need not have a font size, but a written word does). Interestingly, this parallels discussions in memory circles as to what constitutes context (e.g., Chalfonte \& Johnson, 1996), where some researchers would consider font size as context and others would not. For present purposes, we take the more conservative stance that context involves extra-item features and use this definition in this paper. Insofar as contextual features are necessarily separable from the study item (or involve different objects), it should be reasonable to consider the physical context in which an item occurs as an extrinsic cue, or, if one considers a continuum, at least, as more extrinsic than such cues as font size. Although contextual cues are typically studied with respect to how their reinstatement can benefit memory for target items (e.g., McGeoch, 1942; Tulving \& Thomson, 1973), we examine only whether contextual manipulations during study can affect JOLs in ways similar to manipulations made to target items (e.g., size effects). In this literature, environmental context is referred to as study (or test) conditions and may thus align well with Koriat's definition of extrinsic cues as "conditions of learning" (1997).

To date, in JOL studies, manipulations of physical features have largely pertained to study items. Indeed, many findings akin to the font size effect (e.g., Rhodes \& Castel, 2008) have emerged from such manipulations as inverting, masking, or blurring words, (Besken \& Mulligan, 2013; Sungkhasettee, Friedman, \& Castel, 2011; Yue, Castel, \& Bjork, 2013), decreasing the size of objects or faces (Undorf, Zimdahl, \& Bernstein, 2017), deleting portions of images (Besken, 2016), and using less legible text passages (Pieger, Mengelkamp, \& Bannert, 2016). We know of just one study in which physical context was manipulated. Specifically, Saenz and Smith (2018) had participants give JOLs for paired-associate items that overlaid either previously encountered audiovisual scenes or new ones (e.g., a video of an escalator). The new contexts received lower JOLs, but were not remembered better, indicating that metamemory illusions may come from sources other than the study item itself. Although this old-new context effect on JOLs held in a between-subjects manipulation, other studies that have manipulated font size or legibility have found that such effects occur only in withinsubject manipulations, underscoring the importance of relative differences in eliciting JOLs (e.g., Susser, Mulligan, and
Besken, 2013; Magreehan, Serra, Schwartz, \& Narciss, 2016). Such studies indicate an important role for list composition, which may also be thought of as context, in JOLs. Thus, one purpose of this study was to examine whether physical manipulations to context (in the form of backgrounds) analogous to such manipulations as font size might also impact JOLs.

Another purpose of this study was to examine whether manipulations to context might interact with similar manipulations to study items. Currently, one important goal of metacognition research is to delineate how multiple cues are integrated in JOLs (e.g., Rhodes, 2016; Undorf, Söllner, \& Bröder, 2018). Additive cuing (i.e., one cue does not influence how another cue is utilized) has been suggested by Undorf et al. (2018) who found that participants tended to simultaneously give higher JOLs to large words, repeated words, concrete words, and emotional words, but the extent to which each individual cue was utilized was not altered by any of the other cues. However, interactive cuing (i.e., one cue can influence the extent to which another cue is utilized) has been suggested by other studies. For instance, typical font size effects seemed to disappear when there was also a second cue such as items with alternating-case letters (i.e., capitalization) (Rhodes \& Castel, 2008) or non-word items (Mueller, Dunlosky, Tauber, \& Rhodes, 2014). Thus, the inclusion of one cue appeared to have overshadowed the utilization of font size as a cue. Nevertheless, without reporting cue utilization at the individual level, it is perhaps unclear from these studies what sort of cue integration took place. For example, the words with alternating-case letters could have prompted participants to ignore the cue of font size, to no longer use the cue of font size to the same extent (either because fewer individuals experienced the font size effect and/or the font size effect they did experience was much reduced), or to use the cue of font size differently (e.g., a subset of participants who experienced the typical font size effect might have regarded small words in alternating-case letters as more memorable). Here, we employed individual differences analyses to potentially gain a more complete understanding of cue utilization and integration.

In one recent study involving physical cues, Peynircioğlu and Tatz (2019) found that participants gave higher JOLs to large/loud items than to loud/small or large/quiet items, which in turn received higher JOLs than small/quiet items. Interestingly, in this case, modality information appeared to affect JOLs indirectly in that - although large items (i.e., visually intense) were not treated differently from loud items (i.e., auditorily intense) - the judgments reflected intensity information combined across modalities. By indirectly, we mean that a given cue can modulate how another cue (or cues) influences JOLs. A more simplified case is that of list-composition, in which relative differences are required in order for font size to influence JOLs (e.g., Susser, Mulligan, \& Besken, 
2013). Of course, such indirect influences may be more subtle than in these examples. In the current study we explored whether the inclusion of specific contextual information as a cue (e.g., size-varying backgrounds) would alter the use of item-specific information as a cue (e.g., font size).

In Experiment 1, we varied the font size of the words (Rhodes \& Castel, 2008) and surrounded those words with backgrounds that also featured size differences. In Experiment 2, we varied a different physical feature and blurred some of the words (Yue et al., 2013) while similarly blurring some of the backgrounds that surrounded those words. Experiments $3 \mathrm{a}$ and $3 \mathrm{~b}$ served to verify that the reduced font-size and blur effects observed in Experiments 1 and 2 were due to the inclusion of the contextual information rather than any other extraneous factors. In Experiment 4, participants again encountered words and backgrounds that varied in size but also encountered words without backgrounds to enable relative comparisons regarding background presence and examine whether the context-induced reductions in font size remained. In Experiment 5, we varied the font size of the words and surrounded them with backgrounds that varied in terms of either size or blur so as to determine whether the reduced effects that item-specific cues had on JOLs were because they varied in similar ways to the contextual cues or not.

\section{Experiment 1}

\section{Method}

Participants Twenty-four American University students participated in the study for class credit or $\$ 5$. One additional participant was excluded from all analyses due to a technical error during the procedure. The sample sizes of all experiments were determined by a priori power analyses set to detect small to medium effect sizes (Cohen's $f$ s between .18 and .30) at $\alpha=0.05$ and $80 \%$ power (Faul, Erdfelder, Buchner, \& Lang, 2009).

Materials All stimuli were created and presented using MATLAB 2016a with Psychophysics Toolbox 3 on a Toshiba Satellite computer. The words were 40 nouns (see Appendix A) extracted from the SUBTL frequency norms (Brysbaert \& New, 2009) and normalized for frequency ( $M$ $=15.00, S D=0.60)$, number of letters $(M=5.44, S D=1.06)$, and number of syllables $(M=1.71, S D=0.66)$. They were written in Arial font and in small (18-pt) and large (48-pt) sizes. Each word was white, centered, and contained in a black rectangle with an area of $56.4 \mathrm{~cm}^{2}$. Surrounding these words were colored frames that were also either small, 181.1 $\mathrm{cm}^{2}$ (covering $27 \%$ of the display), or large, $482.9 \mathrm{~cm}^{2}$ (covering $72 \%$ of the visual display), thus reflecting the $8: 3$ aspect ratio of the font-size manipulation. Each frame featured a randomly selected color of red, orange, yellow, green, blue, or violet that varied a fixed amount between $5-25 \%$ from its standard RGB components. The reason for random colored frames was to avoid possible habituation to the backgrounds over successive presentations (e.g., Lange, 2005), and the reason for non-focal colors was to make a subsequent colorrecognition test more challenging. The edges outside the colored frames (corresponding to the outermost frames) were black. For JOL responses, a 100-point graphical user interface (GUI) enabled participants to click/drag the cursor (initially set at the mid-point value of 51) anywhere on each rating screen to indicate the probability of recalling the word later. Pencils and paper were used for the distractor and recall tasks.

Design and Procedure Participants studied and provided immediate JOLs for a total of 40 words, with the first and last two acting as primacy and recency buffers. One-quarter of these 36 target words corresponded to each of the four possible pairings based on font size (small, large) and background size (small, large). The words were presented in a different random order to each participant. On each trial, one of the colors was randomly selected for the background frame. The order in which the stimulus conditions were presented was counterbalanced across four groups of participants such that, across participants, all conditions appeared in all presentation slots equally often, within the constraint that no condition appeared more than three times in a row. All participants were tested individually. On-screen instructions informed them of size variations in words, the need to make JOLs for a later free-recall test, how to use the rating scale, and of the size-varying color frames that would appear around the words. Participants were not told why the colors were included or of the upcoming color recognition test. We should note that it was important for participants to know that there would be a free-recall test on the words before the study phase to avoid any misimpression that backgrounds might somehow be relevant to the memory task. Each stimulus was studied for $5 \mathrm{~s}$. Then, a new screen appeared containing the rating slider. Participants had $5 \mathrm{~s}$ to make their JOL ratings, although the rating-scale text disappeared (but not the slider or the JOL rating) after $4 \mathrm{~s}$ to signal that the next study word was about to appear. Two practice trials with the word "sample" (one in large font/small frame, the other in small font/large frame) familiarized them with the procedure.

After the final JOL, a 5-minute distractor task followed in which participants listed as many of the 50 United States and as many different countries as possible. Participants were then given a 5-min free-recall task in which they wrote as many of the studied words as possible. Lastly, participants were given a surprise three-alternative forced choice (3AFC) recognition task on the background color frames presented in the form of squares in a horizontal line up. The purpose was to see 
whether the colors and hence the frames had been paid attention to. One of the lures was the appropriate focal color (e.g., red) and the other varied from the focal red in the same ratio as the color that had actually been seen but in the opposite direction (e.g., participants saw a dark red, so the second lure was a light red). The testing order was the same for all participants but the positions of the three alternatives for each color were random.

\section{Results}

The results are shown in Table 1 (see also Appendix B for detailed results from the background recognition tests). Mean JOLs and percent recall were analyzed using a 2 (Font size: small or large) x 2 (Colored frame size: small or large) repeated measures analysis of variance (ANOVA), with font size corresponding to the item-specific cue manipulation and frame size corresponding to the contextual cue manipulation.

JOL There was a main effect of font size, with words in large font receiving higher ratings than those in small font, $F(1,23)$ $=13.76, M S E=110.3, p<.001, \eta_{p}^{2}=.37$, but no main effect of frame size, $F<1$. There was also an interaction between font size and frame size, $F(1,23)=4.86, M S E=43.77, p=.04, \eta_{p}^{2}$ $=.17$. Because the purpose of this experiment was to compare the addition of frames to that of the typical font size effect (e.g., Rhodes \& Castel, 2008), we used simple effects analyses to further understand how each specific frame type might have affected the font size effect. Simple effects indicated that the font size effect was not as large in the presence of a small frame, $F(1,23)=6.79, M S E=43.69, p=.02, \eta_{p}^{2}=.23$, as it was in the presence of a large frame, $F(1,23)=12.99, M S E=$ $110.3, p=.001, \eta_{p}^{2}=.36$.

Recall There were no main effects of font size, $F<1$, or frame size, $F(1,23)=1.94, M S E=264.8, p=.18$. There was also no interaction, $F(1,23)=2.58, M S E=161.7, p=.12$.
Accuracy Due to the limited number of items recalled in each condition, accuracy was scored first by adding the number of recalled items for which JOLs were $\geq 51$ (the upper half of the scale) to the number of unrecalled items for which JOLs were $<51$. Then, subtracted from this total was the number of recalled items for which JOLs were $<51$ added to the number of unrecalled items where JOLs were $\geq 51$ (e.g., Peynircioğlu \& Tatz, 2019). There was a main effect of font size, $F(1,23)=5.10, M S E=6.87$, $p=.03, \eta_{p}^{2}=.18$, with more accurate JOLs for small- rather than large-font words, as is characteristic of the font size effect (e.g., Rhodes \& Castel, 2008). There was no main effect of frame size, $F<1$ or an interaction, $F(1,23)=1.47, M S E=$ $3.43, p=.24$.

Individual Differences Following Undorf et al. (2018), we characterized cue integration according to whether cues were used separately or together. We also examined potential cue interactions (i.e., non-additive combinations of cues) using an interaction term. Participants' individual JOL responses (for each study item) as a function of font size and frame size were submitted to ANOVAs for independent samples and effect sizes were computed using conventional guidelines (small: $\eta_{p}^{2}>.01$, medium: $\eta_{p}^{2}>.06$, large: $\eta_{p}^{2}>.14$, no evidence of effect: $\eta_{p}^{2}<.01$ ) (Cohen, 1988). The results are presented in Table 2. A considerable majority of participants showed the font-size effect. A slight majority showed interactive cuing, largely consistent with the group-level interaction.

\section{Conclusions}

The font size effect (Rhodes \& Castel, 2008) persisted in the presence of size-varying contextual cues. The JOLs were higher for large items, but recall did not differ. We did not find evidence for a direct effect of background size on JOLs. We did, however, find two potential sources for an indirect influence of background

Table 1 Mean JOLs (1-100), percent recall, and accuracy (-9 to +9) as a function of font size and, if applicable, frame size for the target items in Experiments 1 and 3a. Standard deviations are in parentheses.

\begin{tabular}{|c|c|c|c|c|c|c|}
\hline & \multicolumn{4}{|l|}{ Experiment 1} & \multicolumn{2}{|c|}{ Experiment $3 \mathrm{a}$} \\
\hline & \multicolumn{2}{|l|}{ Small Frame } & \multicolumn{2}{|c|}{ Large Frame } & \multicolumn{2}{|l|}{ No Frame } \\
\hline & Small Font & Large Font & Small Font & Large Font & Small Font & Large Font \\
\hline $\mathrm{JOL}$ & $50.1(11.2)$ & $55.1(10.8)$ & 48.7 (11.0) & $59.6(11.2)$ & $49.9(13.3)$ & $60.4(12.1)$ \\
\hline$\%$ Recall & $23.6(14.4)$ & $19.9(15.7)$ & $24.1(13.8)$ & $28.7(19.4)$ & $25.9(11.1)$ & $28.5(15.3)$ \\
\hline Accuracy $^{\mathrm{a}}$ & $4.3(2.3)$ & $3.5(1.8)$ & $4.7(1.8)$ & $3.0(2.2)$ & $1.6(3.0)$ & $-0.6(3.1)$ \\
\hline
\end{tabular}

${ }^{\text {a }}$ Accuracy scores in Experiment 3a were rescaled (from -18 to +18 ) to better foster comparison with Experiment 1. 
Table 2 The number and proportion of participants showing a given type of cue utilization in each experiment (Expt.) based on the factors manipulated. We also indicate the number and proportion of participants whose individual results are consistent with group-level effects.

\begin{tabular}{|c|c|c|c|}
\hline Expt. & Factors & Type of Cue Utilization ${ }^{a}$ & Relation to Group-Level Effects \\
\hline 1 & $\begin{array}{l}\text { Font size: } \\
\text { (Small, Large) } \\
\text { Frame size: } \\
\quad \text { (Small, Large) }\end{array}$ & $\begin{array}{l}\cdot 1(4.2 \%) \text { no evidence } \\
\cdot 3(12.5 \%) \text { font size only } \\
\cdot 3(12.5 \%) \text { frame size only } \\
\cdot 3(16.7 \%) \text { both additive } \\
\cdot 13(54.2 \%) \text { interactive }\end{array}$ & $\begin{array}{l}\text { - } 17(70.83 \%) \text { showed font size effect (i.e., large words }>\text { small words) } \\
\text { - } 10(41.67 \%) \text { showed larger effects of font size in large backgrounds } \\
\text { compared to small backgrounds }\end{array}$ \\
\hline 2 & $\begin{array}{l}\text { Word blur: } \\
\text { (Blurred, Clear) } \\
\text { Scene blur: } \\
\text { (Blurred, Clear) }\end{array}$ & $\begin{array}{l}\cdot 3(9.4 \%) \text { no evidence } \\
\cdot 2(6.3 \%) \text { word blur only } \\
\cdot 2(6.3 \%) \text { scene blur only } \\
\cdot 7(21.9 \%) \text { both additive } \\
\cdot 18(56.3 \%) \text { interactive }\end{array}$ & $\begin{array}{l}-15(46.9 \%) \text { showed blur effect (i.e., clear words }>\text { blurred words), } \\
\text { which was marginal at the group-level } \\
\text { - } 21(65.6 \%) \text { gave higher JOLs to words in clear scenes than those in blurred ones }\end{array}$ \\
\hline $3 a$ & $\begin{array}{l}\text { Font size: } \\
\text { (Small, Large) }\end{array}$ & $\begin{array}{l}\text { - } 4(16.7 \%) \text { no evidence } \\
\text { - } 20(83.3 \%) \text { font size }\end{array}$ & - $20(83.3 \%)$ showed font size effect \\
\hline $3 b$ & $\begin{array}{l}\text { Word blur: } \\
\text { (Blurred, Clear) }\end{array}$ & $\begin{array}{l}\text { - } 7(29.2 \%) \text { no evidence } \\
\text { - } 17(70.8 \%) \text { word blur }\end{array}$ & - $14(58.3 \%)$ showed blur effect \\
\hline 4 & $\begin{array}{l}\text { Font size: } \\
\text { (Small, Large) } \\
\text { Scene: } \\
\text { (None, Small, Large) }\end{array}$ & $\begin{array}{l}\text { - } 1(2.8 \%) \text { no evidence } \\
\cdot 1(2.8 \%) \text { scene only } \\
\cdot 1(2.8 \%) \text { both additive } \\
\text { - } 33(91.7 \%) \text { interactive }\end{array}$ & $\begin{array}{l}\cdot 24(66.7 \%) \text { showed font size effect } \\
\cdot 20(55.6 \%) \text { gave higher JOLs to words with scenes (regardless of size) } \\
\text { than those without } \\
\cdot 22(61.1 \%) \text { showed reduced font size effect when backgrounds present }\end{array}$ \\
\hline 5 & $\begin{array}{l}\text { Font size: } \\
\text { (Small, Large) } \\
\text { Scene size: } \\
\text { (Small, Large) } \\
\text { Scene blur: } \\
\quad \text { (Blurred, Clear) }\end{array}$ & $\begin{array}{l}\text { - } 1(3.1 \%) \text { all three cues additively } \\
\text { - } 31(96.9 \%) \text { interactive }\end{array}$ & $\begin{array}{l}\cdot 18(56.3 \%) \text { showed font size effect } \\
\text { - } 17(53.1 \%) \text { gave higher JOLs to words in clear scenes than those in blurred ones }\end{array}$ \\
\hline
\end{tabular}

${ }^{\text {a }}$ Please note that the proportions may exceed 100 due to rounding. Cue utilization is classified based on the results of an ANOVA for independent samples (where $\eta_{p}^{2}>.01$ was indicative of cue use).

size on JOLs for font size. First, the font size effect was smaller when the colored frames were small. Moreover, this interaction at the group level appeared to be the result of interactive cuing at the individual level, although we note that not even a majority of participants showed a pattern consistent with this outcome. Second, the font size effect in both types of backgrounds appeared smaller than has been typically reported (Luna, Martín-Luengo, \& Albuquerque, 2017).

\section{Experiment 2}

In Experiment 2, we used a different cue manipulation, namely a specific amount of blur that had been shown to produce an effect analogous to the font size effect (Yue et al., 2013). We also used nature scenes, which are more complex visually and carry more semantic meaning (cf. Davenport \& Potter, 2004) as backgrounds. The purpose was to increase their likelihood of drawing attention, and thus possibly increase the likelihood of eliciting a direct influence of the irrelevant contextual manipulation on JOLs. We also wanted to see whether an indirect influence would emerge regardless, replicating the results of Experiment 1.

\section{Method}

Participants Thirty-two American University students participated in the study for class credit or $\$ 5$. None had participated in Experiment 1. One additional participant was excluded due to not following instructions.

Materials The words were the same 40 nouns used in Experiment 1, written in white, 32-pt. Arial font, centered, and encapsulated by a $56.4 \mathrm{~cm}^{2}$ black rectangle. To generate a blurred version of each word, GIMP, an image manipulation program provided by GNU, was used to introduce a $10 \%$ Gaussian blur to its pixels (Rosner et al., 2015). The black rectangle containing each word was surrounded by a nature scene that covered the entire rest of the display. These nature scenes were obtained from a Google image search for "nature OR scene OR landscape" with additional filters of "free and for commercial use" and were in .jpg format with $1600 \times 1000$ or greater resolution. To maintain uniformity across the background stimuli, further constraints were that humans or manmade objects were not present, no reflections were present in bodies of water (to avoid any initial blur), and file sizes were greater than 1.0 MB to ensure high quality. A total of 80 images were included in the study, 40 of which were used as backgrounds for the target words during the study phase, and 
40 of which were used as lures during the recognition test phase of the backgrounds. As with the words, $10 \%$ Gaussian blur was introduced to the images using the same program.

Design and Procedure The design was similar to that of Experiment 1 except that blur was manipulated instead of size, and each background was unique. Stimulus conditions included blurred word/blurred scene, blurred word/clear scene, clear word/blurred scene, and clear word/clear scene. As with Experiment 1, the words and images were presented in separate random orders for each participant, but the presentation order of the conditions was counterbalanced across four groups of participants. The presentation order of the backgrounds was separately randomized for each participant in the surprise recognition test, and the backgrounds used as contexts in the study phase were interchanged with the backgrounds used as lure backgrounds in the recognition test phase across two subgroups of participants. The procedure was also similar to that of Experiment 1, except that a free-choice recognition test (using the " $y$ " or " $n$ " button) was used to test memory for the backgrounds, and all backgrounds were presented in their clear versions.

\section{Results}

The results are shown in Table 3. We analyzed mean JOLs, percent recall, and accuracy for words, and sensitivity (d') and response bias $(\mathrm{C})$ for incidental nature-scene recognition, using 2 (Word: blurred, clear) x 2 (Nature scene: blurred, clear) repeated measures ANOVAs. See Appendix B for the details of background nature-scene recognition.

JOL There was an effect approaching significance for word blur in the expected direction, $F(1,31)=3.71, M S E=$ 175.7, $p=.06, \eta_{p}^{2}=.11$, with blurred words receiving lower JOLs than clear words. This time, there was also a main effect of scene blur, $F(1,31)=17.08, \operatorname{MSE}=77.59, p<.001, \eta_{p}^{2}=$
.36 , with words presented in the context of blurred scenes receiving lower JOLs than words presented in the context of clear scenes. There was no interaction, $F<1$.

Recall There were no main effects of word blur or scene blur, $F_{\mathrm{s}}$ $<1$, but there was an interaction between the two, $F(1,31)=$ $5.57, M S E=177.2, p=.02, \eta_{p}^{2}=.15$. Blurred words appeared to be recalled best when the scene was also blurred, whereas clear words appeared to be recalled best when the scene was also clear. Simple effects analyses indicated a marginal difference in recall in which blurred words were recalled more often than clear words in the presence of blurred scenes, $F(1,31)=3.42$, $M S E=163.2, p=.07, \eta_{p}^{2}=.10$; however, no difference was observed between blurred and clear words in the presence of clear scenes, $F(1,31)=2.10, M S E=207.0, p=.15$.

Accuracy Accuracy scores were computed as in the previous experiment. There was no main effect of word blur, $F<1$, but there was a main effect of scene blur, $F(1,31)=4.89, M S E=$ $4.16, p=.03, \eta_{p}^{2}=.14$, with better accuracy for words in blurred relative to clear backgrounds. There was no interaction, $F<1$.

Individual Differences The analyses were similar to those of Experiment 1. The results are presented in Table 2. A considerable majority of participants showed the effect of background blur consistent with the group-level main effect. Nearly half of the participants showed evidence for an effect of word blur, an effect that approached significance at the group-level. Although no group-level interaction was found, most individuals used cues interactively.

\section{Conclusions}

The present experiment appeared to replicate the blur effect, but, again, with a markedly reduced JOL effect size compared to those reported in the literature (Yue et al., 2013). Also, a

Table 3 Mean JOLs, percent recall, and accuarcy for the target items, and d' and C measures for old/new nature scene recognition, as a function of Word Blur and, if applicable, Scene Blur in Experiments 2 and 3b. Standard deviations are in parentheses.

\begin{tabular}{|c|c|c|c|c|c|c|}
\hline & \multicolumn{4}{|l|}{ Experiment 2} & \multicolumn{2}{|l|}{ Experiment $3 b$} \\
\hline & \multicolumn{2}{|l|}{ Blurred Scene } & \multicolumn{2}{|l|}{ Clear Scene } & \multicolumn{2}{|l|}{ No Scene } \\
\hline & Blurred Word & Clear Word & Blurred Word & Clear Word & Blurred Word & Clear Word \\
\hline $\mathrm{JOL}$ & $44.9(15.9)$ & 48.7 (18.4) & $50.7(15.3)$ & $56.0(17.3)$ & $52.1(18.4)$ & $61.1(22.7)$ \\
\hline$\%$ Recall & $25.0(17.8)$ & $19.1(15.0)$ & $20.8(14.6)$ & $26.0(16.8)$ & $13.0(9.1)$ & $15.0(9.0)$ \\
\hline Accuracy $^{\mathrm{a}}$ & $4.4(2.4)$ & $4.9(2.1)$ & $3.8(1.8)$ & $3.9(2.5)$ & $1.5(4.3)$ & $-0.9(4.4)$ \\
\hline $\mathrm{d}^{\prime}$ & $.52(.50)$ & $.41(.52)$ & $.86(.56)$ & $.79(.61)$ & -- & -- \\
\hline $\mathrm{C}$ & $.30(.36)$ & $.36(.36)$ & $.13(.32)$ & $.20(.34)$ & -- & -- \\
\hline
\end{tabular}

${ }^{\text {a }}$ Accuracy scores in Experiment $3 \mathrm{~b}$ were rescaled (from -18 to +18 ) to better foster comparison with Experiment 2. 
large effect was observed for the contextual manipulation of blur; participants predicted that words in clear scenes would be more memorable than those in blurred scenes. This prediction was illusory because words in clear scenes were not recalled better than those in blurred scenes. These results also contrasted the typically greater effects observed for intrinsic cues with respect to cue utilization (Koriat, 1997), possibly because, unlike the colored frames of Experiment 1, the more unique nature-scene backgrounds captured participants' attention and prompted background blur to be utilized as a cue even when it was not relevant to where participants' attention ought to have been directed (i.e., the words).

Interestingly, most participants seemed to use the cues of word blur and background blur interactively even though an interaction was not found at the group level-likely because individuals' particular patterns of cue utilization were more varied than in Experiment 1. At the same time, there was also some evidence for an indirect influence of background cues on JOLs (as in Experiment 1), indicated by a reduction of the blur effect obtained previously (Yue et al., 2013). That is, although the effect of background blur did not interact with the effect of word blur on JOLs at the group level, the replication of an overall reduction in the effect of item-specific cues, in this case word blur, suggests that context manipulations could indeed be the reason. Nevertheless, minor methodological deviations from Rhodes and Castel (2008) and Yue et al. (2013) prohibit us from firmly concluding that the observed effect based on intrinsic cues was smaller than those reported previously. Experiments $3 \mathrm{a}$ and $3 \mathrm{~b}$ served as control experiments to see if the magnitude of the illusions with the item cues returned to typical levels if there were no contextual cues.

\section{Experiments $3 a$ and $3 b$}

Aside from the inclusion of colored frames, Experiment 1 differed from Rhodes and Castel's (2008) first experiment in some minor ways: we used a different list of nouns, participants provided their JOLs on a rating slider instead of on paper, and two words were used as primacy and recency buffers (instead of three apiece). Aside from the inclusion of nature scenes, the method for Experiment 2 differed from Yue et al. (2013) in that their participants viewed four consecutive lists each composed of 26 different words in 44-pt font (we used 32-pt), had only one primacy and one recency buffer word per list (we used two in our single list), and engaged in 10-s distractor tasks prior to free recall. The following two experiments were methodologically identical to Experiments 1 and 2 except that no backgrounds were present.

\section{Experiment 3a}

Method Twenty-four American University students participated in the study for class credit or $\$ 5$. None had participated in the previous experiments. Data from two additional participants were excluded because their JOLs were over 3 SDs from the mean in one or more conditions (no statistical outcomes differed as a result of their exclusion), and another participant was excluded because he/she did not follow instructions. The method was identical to that of Experiment 1 except that during the study/JOL phase no colored frames surrounded the words.

Results The results are presented in Table 1 (for individual differences, see Table 2). JOLs were higher for words presented in large font, $F(1,23)=31.9, M S E=41.65, p<.01, \eta_{p}^{2}=$ .58 , but recall did not differ as a function of font size, $F<1$. Accuracy scores for words in small font were higher, $F(1,23)$ $=13.94, M S E=17.43, p<.01, \eta_{p}^{2}=.38$. Thus, the font size effect was no longer unusually small (cf. Rhodes and Castel, 2008). Indeed, when compared to that in Experiment 1, the JOL effect increased by about $50 \%$ overall.

\section{Experiment 3b}

Method Twenty-four American University students participated in the study for class credit or $\$ 5$. None had participated in the previous experiments. The method was the same as that of Experiment 2 except that no nature scenes surrounded the words during the study/JOL phase.

Results The results are presented in Table 3 (for individual differences, see Table 2). JOLs were higher for clear items, $F(1,23)=7.35, M S E=974.4, p=.01, \eta_{p}^{2}=.24$, but recall did not differ as a function of blur, $F<1$. Accuracy scores for blurred words were higher, $F(1,23)=8.35, M S E=266.0, p<$ $.01, \eta_{p}^{2}=.26$. Thus, the blur effect was no longer unusually small (cf. Yue, Castel, \& Bjork, 2013). Indeed, when compared to that in Experiment 2, the JOL effect increased by close to $60 \%$.

\section{Conclusions}

We surmised that the reductions in JOL effect sizes for both item-specific cue manipulations were due to the addition of backgrounds. That is, background manipulations likely influenced how item-specific cues were utilized by compressing the JOL differences observed in typical experiments that do not have background manipulations. This appeared to be true both when the manipulation of frame size as the background did not directly produce a reliable difference in JOLs for study items (Experiment 1) as well as when the manipulation of 
nature-scene blur did directly produce such a difference (Experiment 2). Also, because the font size and blur of words did not affect memory performance, these reductions may represent reductions in the illusions themselves. At the individual level, fewer participants showed evidence for both effects (about 10\% fewer in either case) and fewer participants showed large effects of either, possibly because the more complex cue environment prompted less extreme JOL responses. Of course, comparisons across experiments require caution, and future experiments are needed to ensure that the current results were not simply due to other differences between the experiments.

\section{Experiment 4}

Varying the size of solid-colored backgrounds failed to influence JOLs directly (Experiment 1) whereas varying the blur of nature scenes did (Experiment 2). One possibility was that the nature scenes were more attention-capturing, prompting the utilization of the contextual cue in JOLs. If so, varying nature scenes in terms of size (as well as the study items) should result in the utilization of the contextual size cue, as well. Another possibility was that the words with clear backgrounds in Experiment 2 were regarded as more memorable because of the greater detail those backgrounds contained (cf. Davenport \& Potter, 2004) - with blur reducing their identity as nature scenes. If so, although a background size manipulation with nature scenes might not influence JOLs directly (as in Experiment 1), higher JOLs should be expected for words with backgrounds than those without. Further, given that comparisons across experiments require caution, the present experiment also allowed us to examine whether the context-induced font-size reduction observed between experiments would replicate within a single, mixed-list design.

\section{Method}

Participants Thirty-six American University students participated in the study for course credit. None had participated in previous experiments.

Materials The words were 42 nouns extracted from the SUBTL frequency norms. All but two additional words ("booth" and "clerk") were in common with previous word lists. The backgrounds were 72 randomly selected nature scenes from the 80 used in Experiment 2. The nature scenes occupied either a small or a large frame surrounding the words whose dimensions corresponded to those in Experiment 1.
Design and Procedure The design of this experiment was similar to that of Experiment 1 except that additional conditions included small and large words without backgrounds, and the backgrounds varying in size included nature scenes rather than solid colors. Thus, stimulus conditions included small word/no scene, small word/ small scene, small word/large scene, large word/no scene, large word/small scene, and large word/large scene. The presentation order of these conditions was counterbalanced across six groups of participants. The design and procedure for the background recognition test was similar to that of Experiment 2 except that, if a scene was presented as a background during the study phase, its presentation format (small or large) at test remained the same. Likewise, half of the lure backgrounds were small, and half were large.

\section{Results}

The results are shown in Table 4. Response variables included mean JOLs and percent recall. Our two research questions lent themselves to two separate analyses. To address the effect of nature-scene size, results were analyzed using 2 (Font size: small, large) x 2 (Nature scene: small, large) ANOVAs. To address the general effect of adding context, results were analyzed using 2 (Font size: small, large) x 2 (Background: absent, present) repeated measures ANOVAs. Thus, in the background-present conditions, we collapsed responses across nature-scene size. Additional response variables for the first set of analyses (pertaining to nature-scene size) included $\mathrm{d}^{\prime}$ and $\mathrm{C}$ for the background recognition task, and the details can be found in Appendix B.

Nature-Scene Size First, we examined the effect of naturescene size. For JOLs, there was a main effect of font size, $F(1,35)=19.29, M S E=77.26, p<.001, \eta_{p}^{2}=.36$, in which words in large font received higher JOLs. There was no main effect of nature-scene size nor an interaction of font size and nature-scene size, $F s<1$. For percent recall, there were no main effects, nor was there an interaction, $F_{S}<1$.

Effect of Context Next, we examined the general effect of adding backgrounds. For JOLs, there was a main effect of font size, $F(1,35)=39.33, M S E=63.46, p<.001, \eta_{p}^{2}=.53$, in which large words received higher JOLs than small words. There was also a main effect of background presence, $F(1$, $35)=8.00, M S E=136.94, p=.008, \eta_{p}^{2}=.19$, in which words with backgrounds received higher JOLs than those without. Most importantly, there was an interaction, $F(1,35)=4.36$, $M S E=29.54, p=.04, \eta_{p}^{2}=.11$. Simple effects analyses indicated that the font size difference was larger when context was absent, $F(1,35)=34.57, M S E=54.36, p<.001, \eta_{p}^{2}=.50$, than 
Table 4 Mean JOLs, percent recall, and accuracy $(-6$ to +6$)$ for the target items, and d' and C measures for old/new nature scene recognition, as a function of font size and scene size in Experiment 4. Standard deviations are in parentheses.

\begin{tabular}{|c|c|c|c|c|c|c|}
\hline & \multicolumn{2}{|l|}{ Small Scene } & \multicolumn{2}{|l|}{ Large Scene } & \multicolumn{2}{|l|}{ No Scene } \\
\hline & Small Font & Large Font & Small Font & Large Font & Small Font & Large Font \\
\hline JOL & $48.4(12.7)$ & $54.0(11.3)$ & $47.9(8.7)$ & $55.2(10.2)$ & $41.6(14.4)$ & $51.0(13.0)$ \\
\hline$\%$ Recall & $27.3(17.4)$ & $27.3(17.0)$ & $25.5(15.7)$ & $27.3(20.4)$ & $25.9(20.9)$ & $21.3(20.9)$ \\
\hline Accuracy & $1.6(2.6)$ & $0.1(2.6)$ & $1.6(2.7)$ & $0.2(3.0)$ & $2.2(3.0)$ & $1.06(3.1)$ \\
\hline $\mathrm{d}^{\prime}$ & $.82(.73)$ & $.96(.89)$ & $.97(1.0)$ & $.95(.86)$ & -- & -- \\
\hline $\mathrm{C}$ & $.42(.39)$ & $.36(.40)$ & $.35(.45)$ & $.36(.44)$ & -- & -- \\
\hline
\end{tabular}

when context was present, $F(1,35)=19.29, M S E=38.63, p<$ $.001, \eta_{p}^{2}=.36$. Thus, the inclusion of context reduced the effect of font size on JOLs by roughly $30 \%$. For percent recall, there were no main effects of font size, $F<1$, or of background presence, $F(1,35)=1.72, M S E=219.34, p=.20$. Nor was there an interaction between font size and background presence, $F(1,35)=1.71, M S E=162.69, p=.20$. For JOL accuracy, there was a main effect of font size, $F(1$, $35)=9.36, M S E=6.28, p=.004, \eta_{p}^{2}=.21$, in which words in small font received higher accuracy scores. There was no main effect of background presence, $F(1,35)=2.76, M S E=7.35, p$ $=.11$. There was no font size by background presence interaction, $F<1$.

Individual Differences Individual differences were assessed by submitting individual JOL responses to 2 (Font Size: Small, Large) x 3 (Background: None, Small, Large) ANOVAs for independent samples. The results are presented in Table 2. A considerable majority of participants showed the font-size effect as well as interactive cuing. Of those showing interactive cuing, most individual patterns were consistent with the group-level interaction.

\section{Conclusions}

Although we manipulated the size of background nature scenes, it did not appear to be utilized as a cue. Instead, we found that words in any nature scene (regardless of size) received higher JOLs than those that were not surrounded by backgrounds. The outcome that background presence did influence JOLs would appear similar to that of Experiment 2, in which words in clear nature scenes received higher JOLs than those in blurred nature scenes (where presumably, blur interfered with the backgrounds' identity as meaningful scenes). Conversely, perhaps manipulating background size failed to influence JOLs because both small and large scenes attracted similar amounts of attention and depicted the same content. We should also note that we failed to find an interaction in which the font size effect varied as a function of nature-scene size. This contrasts Experiment 1, in which we found an interaction where the font size effect was more reduced in small relative to large solid-colored frames.

Consistent with the results from previous experiments, the presence of a background reduced the font size effect and did so when the words were presented with and without backgrounds in the same list. Thus, we replicated our findings across Experiments 2 and $3 \mathrm{~b}$ in showing that, even as background cues can directly influence JOLs, they can also influence JOLs indirectly by reducing the extent to which item cues are used. Regarding the intrinsic and extrinsic cue distinction in Cue Utilization Theory (Koriat, 1997), it is worth noting that the intrinsic cue of font size was more influential on JOLs than the direct effect produced by the extrinsic cue of background presence. Although consistent with typical cue utilization, this contrasts the results from Experiment 2, in which the direct influence of background blur (extrinsic) was more influential than that of word blur (intrinsic).

Also, participants did not recall words differently based on the presence or absence of nature scenes; thus, their JOL prediction that words in nature scenes would be more memorable was illusory. This parallels the finding for clear vs. blurred nature scenes in Experiment 2 in suggesting that participants may predict contextual details to benefit memory, even though actual performance may not differ. As mentioned, seductive details typically harm memory performance (e.g., Mayer et al., 2008). Nevertheless, one survey of metacognitive beliefs (McCabe, 2011) found that respondents predicted that items learned with additional, but irrelevant details would be better learned. Interestingly, it has been suggested by Benjamin, Bjork, and Schwartz (1998) that inflexible beliefs about memory may result in an invalid use of cues in JOLs (e.g., using retrieval fluency from semantic memory to predict performance on a test of episodic memory). Similarly, we note that perhaps inflexible or misapplied beliefs about context might explain why background cues were utilized, but not validly, in our experiments. That is, although participants were instructed that they would be engaging in free recall of only the words, that they were to provide JOLs only for the words, and that 
contexts were not relevant to the memory task as well as not distinctive from each other, their beliefs might have been based on knowledge/experience of the usually beneficial effects of context reinstatement or use of context in other memory scenarios.

\section{Experiment 5}

In our previous experiments, the contextual manipulations of background size and blur were accompanied by corresponding item-specific manipulations of size and blur, respectively. Across experiments, we found that the addition of backgrounds reduced the extent to which item-specific cues were utilized. Nevertheless, it remains unclear whether this reduction in cue-utilization is attributable to the correspondence between item-specific and contextual manipulations or not. That is, using similar item-specific and contextual manipulations (e.g., blur) might have drawn attention to and prompted cue utilization of the contextual manipulations, whereas the contextual manipulations might not have influenced JOLs had the correspondence to item-specific cues been weaker. Alternatively, it might be that the details present in the backgrounds themselves are important for cue-utilization, in which case item-specific and contextual cues should be utilized even with different feature manipulations. To address this, in the current experiment we varied font size as an item-specific cue as well as background size (i.e., corresponding) and background blur (i.e., non-corresponding) as two different contextual cues.

\section{Method}

Participants Thirty-two American University students who were fluent in English participated in the study for class credit. None had participated in the previous experiments.

Materials The words were the 42 nouns used in Experiment 4. Because each word was accompanied by a different background in this experiment, we used the 80 nature scenes from Experiment 2 as backgrounds along with two additional nature scenes. Following the same specifications used in previous experiments, the nature scenes occupied either a small or large frame and, at the same time, featured either clear or blurred versions. This time, videos of the study materials and images for the background recognition test were created using MS Powerpoint. Pencils and paper were used for all responses. Stimuli were displayed on a Dynex television set and participants were seated so that the stimuli occupied roughly the same visual angle.

Design and Procedure The design of this experiment was similar to that of Experiment 1 except for the additional stimulus conditions pertaining to background blur. Thus, there were eight possible stimulus conditions corresponding to a 2 (font size) $\times 2$ (scene size) $\times 2$ (scene blur) factorial design. In this experiment, the presentation order of words and their respective scenes was randomly determined and the same for all participants. The order of conditions was also randomly determined within the constraint that a given stimulus level (e.g., large word) was not repeated more than four times in a row, the same for all participants, and counterbalanced across eight groups of participants. Two items (and their respective backgrounds) served as primacy buffers, but we did not include recency buffers because the filler tasks had previously appeared sufficient to eliminate such effects. As in Experiment 4, the background manipulations (e.g., small/ clear) used in the incidental recognition test were kept the same across study and test conditions, and an equal number of lure items featured the same sort of background manipulations. That is, the lure items were also blurred or clear as well as small or large. The procedure followed those of previous experiments except that the participants were tested in groups of up to four people.

\section{Results}

The results are shown in Tables 5 and 6. Response variables included mean JOLs and percent recall for the target words, and sensitivity (d') and response bias (C) measures for naturescene recognition (for detailed results of background recognition, see Appendix B). Response variables for the

Table 5 Mean JOL, percent recall, and accuracy $(-5$ to +5$)$ for the target items as a function of font size, scene size, and scene blur in Experiment 5 . Standard deviations are in parentheses.

\begin{tabular}{|c|c|c|c|c|c|c|c|c|}
\hline & \multicolumn{4}{|c|}{ Blurred Scene } & \multicolumn{4}{|l|}{ Clear Scene } \\
\hline & \multicolumn{2}{|l|}{ Small Scene } & \multicolumn{2}{|l|}{ Large Scene } & \multicolumn{2}{|l|}{ Small Scene } & \multicolumn{2}{|l|}{ Large Scene } \\
\hline & Small Font & Large Font & Small Font & Large Font & Small Font & Large Font & Small Font & Large Font \\
\hline JOL & $46.0(20.5)$ & $51.4(19.7)$ & 43.4 (17.6) & $49.7(17.3)$ & $48.7(18.8)$ & $52.2(17.9)$ & $48.7(19.6)$ & $55.6(17.6)$ \\
\hline Accuracy & $0.2(1.4)$ & $0.2(1.3)$ & $0.0(0.7)$ & $0.1(1.3)$ & $0.1(1.2)$ & $0.3(1.2)$ & $0.2(0.9)$ & $0.5(1.3)$ \\
\hline$\%$ Recall & $22.9(22.3)$ & $18.9(20.0)$ & $17.7(19.3)$ & $21.7(20.2)$ & $24.0(21.0)$ & $26.3(18.6)$ & $19.4(19.1)$ & $19.4(18.5)$ \\
\hline
\end{tabular}


Table 6 Mean d' and C measures for old/new nature scene recognition as a function of scene size and scene blur in Experiment 5. Standard deviations are in parentheses.

\begin{tabular}{lccccc}
\hline & \multicolumn{3}{c}{ Blurred Scene } & & \multicolumn{2}{l}{ Clear Scene } \\
\cline { 2 - 3 } \cline { 5 - 6 } & Small Scene & Large Scene & & Small Scene & Large Scene \\
\hline $\mathrm{d}^{\prime}$ & $.19(.59)$ & $.24(.46)$ & & $.23(.57)$ & $.70(.55)$ \\
$\mathrm{C}$ & $.19(.44)$ & $.18(.49)$ & & $.26(.47)$ & $.31(.47)$ \\
\hline
\end{tabular}

target words were analyzed using 2 (Font size: small, large) $\mathrm{x}$ 2 (Background size: small, large) x 2 (Background blur: blurred, clear) repeated measures ANOVAs. Because itemspecific cues had not affected background recognition in previous experiments, only background size and background blur were included as factors for the analyses on recognition data.

JOL and Recall There was a main effect of font size, $F(1,31)=$ 13.34, MSE $=62.74, p<.001, \eta_{p}^{2}=.30$, in which words in large font size received higher JOLs than those in small font size. There was also a main effect of scene blur, $F(1,31)=$ 9.53, $M S E=91.68, p=.005, \eta_{p}^{2}=.24$, in which words presented in the context of clear scenes received higher JOLs than words presented in the context of blurred scenes. None of the other main effects or interactions were significant (Background Size X Background Blur: $F(1,31)=1.90$, $M S E=174.06, p=.18$; otherwise all $F S<1$ ). For percent recall, there were no significant main effects or interactions (Background Size: $F(1,31)=2.88, M S E=312.90, p=.10$; Background Blur: $F(1,31)=1.67, M S E=183.68, p=.21$; Font Size X Background Size: $F(1,31)=1.67, M S E=134.68$, $p=.21$; Font Size X Background Size X Background Blur: $F(1,31)=2.02, M S E=308.87, p=.16$; otherwise all $\left.F_{S}<1\right)$.

Accuracy We did not find a main effect of font size on JOL accuracy, $F(1,31)=1.24, M S E=1.26, p=.27$, nor one of background blur, $F(1,31)=1.00, M S E=1.27, p=.33$. Although inconsistent with our JOL and recall findings, perhaps an effect on accuracy did not emerge because the JOL effects were weaker than in previous experiments or because there were fewer words included in each condition. No other main effects or interactions were significant (Font Size x Background Blur: $F(1,31)=1.41, M S E=1.11, p=.24$; all other $\left.F_{S}<1\right)$.

Individual Differences Individual differences were assessed by submitting individual JOL responses to 2 (Font Size: Small, Large) x 2 (Background Size: Small, Large) x 2 (Background Blur: Small, Large) ANOVAs for independent samples. The results are presented in Table 2. Most individuals showed effects of font size and background blur consistent with the main effects along with idiosyncratic interactive cuing, thereby precluding any group interaction.

\section{Discussion}

Our finding that the font size and background blur effects could occur within a mixed-list design would suggest that having a similar, or corresponding, manipulation between item-specific cues and contextual cues is not necessary for a reduction in cue utilization. In fact, the font size effect in this experiment involving three cues showed the weakest effect. The background blur effect, too, was weaker than in Experiment 2 where the only other cue manipulation had been word blur. Thus, increasing the number of cue manipulations appeared to result in fewer individuals showing either effect as well as possibly fewer extreme expressions of the effects.

\section{General Discussion}

Relative differences in the physical attributes of study items often result in metamemory illusions in which participants predict one sort of item to be more memorable even when it is, in fact, not (e.g., Rhodes \& Castel, 2008). With respect to cue utilization (Koriat, 1997), such intrinsic cues are utilized but ineffectively so. In this study, we examined the influence that similar changes to backgrounds, or extrinsic cues, had on the metamemory illusions originally produced by just intrinsic cue manipulations of font size (e.g., Rhodes \& Castel, 2008) and blur (e.g., Yue et al., 2013). Our findings demonstrated that metamemory illusions may occur with contextual cue manipulations rather than just those specific to features of the study items. In addition, our findings indicated that multiple cues can be utilized in complex and often interactive patterns that may differ across individuals.

According to Cue-Utilization Theory (Koriat, 1997), JOLs are based on heuristic considerations of how various factors present during study might influence later memory performance. Further, Cue-Utilization Theory also suggests that cues may be distinguishable as intrinsic or extrinsic, the latter of which are typically discounted in the formation of JOLs. Although in some situations, findings indicating that intrinsic cues can be discounted relative to extrinsic ones (e.g., Dunlosky \& Matvey, 2001) have perhaps led to a deemphasis in differentiating these cue types, it is still worth considering whether this distinction might be meaningful when cues involve physical attributes during study. That is, unlike more traditional types of cues (e.g., an item's concreteness), cues involving physical attributes are frequently used invalidly in JOLs (some factor is predicted to benefit memory when it does not or does in the opposite way). As such, it is possible that such metamemory illusions would arise only when the cues are intrinsic or would at least show the 
hierarchy where the effect of intrinsic cues on JOLs would be greater than that of their extrinsic counterparts. Across experiments, we found that extrinsic cues pertaining to backgrounds could produce metamemory illusions.

Thus, consistent with findings involving non-physical cues (e.g., Castel, 2008; Dunlosky \& Matvey, 2001; Matvey, Dunlosky, \& Schwartz, 2006), we found mixed support for whether extrinsic cues were utilized less than their intrinsic counterparts using physical cues. With respect to cue utilization, then, what appears to be more important than the intrinsic vs. extrinsic nature of the cues is whether participants deem them to be relevant to memory and hence make use of them as a heuristic. This notion is perhaps more consistent with the analytic processing theory as applied to JOLs (Mueller \& Dunlosky, 2017), which stipulates that JOLs are based on beliefs formulated or reactivated during JOL experiments. With respect to cue validity, we show that, as with intrinsic, or item-specific, cues (e.g., Rhodes \& Castel, 2008), extrinsic, or contextual, cues may also be used in ineffective, or "illusory" ways.

Another aspect of cue utilization that has received some recent attention concerns how cues are integrated in JOLs. Rhodes (2016) notes that much of the literature has focused on how single cues impact JOLs without considering how various cues might together impact JOLs. Although two subsequent investigations on cue integration provided plausible reasons for why their cues might combine interactively, they instead found only additive cue integration (Peynircioğlu \& Tatz, 2019; Undorf et al., 2018). In the present study, we showed that the presence of backgrounds varying in size (Experiments 1, 4, and 5) and blur (Experiments 2 and 5) reduced the extent to which an item's font size (Rhodes \& Castel, 2008) and blur (Yue et al., 2013), respectively, were utilized. Indeed, a random-effects meta-analysis (Del Re, 2015) on the item-specific differences observed in Experiments $1-4$ with and without backgrounds indicated that this reduction was reliable $(Z=2.15, p=.031)$ and a notable reduction in estimated effect size $\left(d=-.33, \mathrm{CI}_{95}\right.$ : $0.62,-0.03)$. In our view, this provides convincing evidence that a given cue might, instead of (or in addition to) directly affecting JOLs, indirectly affect how another type of cue is utilized. These results suggest that cues can combine in dynamic ways in JOLs and perhaps invite more systematic analyses of what factors lead cues to be used differently depending on their larger cue environment.

Indeed, at the individual level, we found that most participants used cues interactively. However, discrepancies in the specific, interactive JOL predictions participants made often prevented the emergence of group-level interactions. Thus, our results were consistent with Undorf et al. (2018) in demonstrating that several cues may be integrated in JOLs but diverged in suggesting that interactive cuing may be the norm. One notable difference between these studies is that our cues all involved "physical features", which one might expect to be more subject to constraints of attentional resources or saliency. In fact, across experiments we found that as the number of competing cues increased, the extent to which individual cues (e.g., font size) were utilized decreased. Although, superficially, this would seem in line with the analytic processing theory (Mueller \& Dunlosky, 2017; Undorf et al., 2018), which would predict an overall reduction in cue utilization with more cues (due to their competition for beliefs), we found that the reduction in JOLs specific to any one cue manipulation (e.g., font size) appeared to be accompanied by a more elaborate and interactive use of the available cues on the part of participants. The finding that participants differed in their interactive predictions may suggest that either participants may be able to form quite complex beliefs online or may rely on non-analytic processes (Undorf et al., 2018). Because these alternatives are not mutually exclusive, increasing the number of competing cues and their attention-capturing properties in future work may lead to a better understanding of cue utilization.

In conclusion, we have demonstrated that physical cues may interact with each other in terms of how they influence JOLs. Specifically, in the present case, the inclusion of backgrounds reduced participants' predictions that large words would be more memorable than small ones (e.g., Rhodes \& Castel, 2008) and that clear words would be more memorable than blurred ones (e.g., Yue et al., 2013). Because these predictions represented metamemory illusions to start with, it can also be said that the font size and blur illusions themselves were reduced. At the individual level, it would seem that as more cues become available, individuals utilize them more interactively, thereby decreasing the influence of any one cue (e.g. font size). Ecologically, this would indicate that such illusions may not be as influential in our day-to-day lives because items that we intend to remember (e.g., a product in an advertisement) typically occur in some sort of context. More generally, the present work shows the promise of studying JOLs in settings more analogous to everyday life (e.g., Rhodes, 2016). And, because cues can be used interactively in predicting memory performance, going forward, perhaps more holistic considerations of how multiple cues may affect JOLs would be more realistic.

Open Practices Statement Materials and data for all experiments are available upon request to the first author. None of the experiments was preregistered.

Author Notes Participant reimbursement was supported by an internal grant to the second author. We thank Bennett L. Schwartz and Emily G. Peterson for helpful comments on an earlier version of this manuscript. We thank Melissa Baney for collecting a portion of the data in Experiment 2. We thank Sara Wong for collecting the data in Experiment 5. 


\section{Appendix A: Target Study Items}

Here, we present the nouns extracted from the SUBTL frequency norms (Brysbaert \& New, 2009) and used as target study items in Experiments 1-5. The last two words in the third column were exclusive to Experiment 4, which required two more words than the other experiments. Except for the recency and primacy buffers, the words were always presented in a random order.

\section{Appendix B: Results from Incidental Background Recognition Tests}

In Experiments 1, 2, 4, and 5, surprise recognition tests were included as indirect measures of attention. Except for the mixed results in Experiment 1, there was clear evidence across experiments that participants indeed paid attention to the backgrounds (as indicated by above chance performance). Results are summarized as follows:

- Experiment 1. Exact binomial tests were used to assess whether the target color was recognized above chance from among two lure items ( $>33.3 \%$ ) in each color category. Sample sizes for these tests varied slightly due to an initial programming error that produced four missing responses (three from one individual). Recognition was above chance for red $(p=.03)$, orange $(p=.01)$, and violet $(p=.006)$ color categories, but not for yellow, green, or blue $(p>.10)$. Despite these limitations, an analysis of JOL responses that included only those trials with red, orange, and violet colored frames did not produce different results from those reported in the text.

- Experiment 2. Signal detection analyses showed a mean hit rate of $52 \%$ and a mean false alarm rate of $29 \%$. The d's were reliably above zero in each condition [Blurred Word/ Blurred Scene: $t(31)=5.81$, Blurred Word/Clear Scene: $t(31)=8.67$, Clear Word/Blurred Scene: $t(31)=4.39$, Clear Word/Clear Scene: $t(31)=7.31$, all $p s<.001]$. There was no effect of word blur on d's, $F(1,31)=1.66$, $M S E=.17, p=.21$, but formerly clear scenes were recognized better than formerly blurred scenes, $F(1,31)=$ 19.37, MSE $=.22, p<.001, \eta_{p}^{2}=.38$. There was no interaction, $F<1$. The Cs indicated a conservative response bias in every condition [Blurred Word/Blurred Scene: $t(31)=4.76$, Blurred Word/Clear Scene: $t(31)=2.29$, Clear Word/Blurred Scene: $t(31)=5.63$, Clear Word/ Clear Scene: $t(31)=3.43$, all $p s<.05]$. There was a slight trend suggesting scenes formerly presented with clear words were responded to more conservatively than those formerly presented with blurred words, $F(1,31)=3.05$, $M S E=.05, p=.09, \eta_{p}^{2}=.09$. Participants responded more conservatively to scenes that were formerly blurred than clear ones, $F(1,31)=16.74, M S E=.06, p<.001, \eta_{p}^{2}=.35$. There was no interaction, $F<1$. Our finding that participants recognized clear scenes better than blurred scenes can be explained by the encoding specificity principle because all scenes were tested in a clear fashion regardless of whether they had been first encountered in a clear or a blurred fashion (Tulving \& Thomson, 1973). Also, such changes at test often result in both lower d's and more conservative responses (Feenan \& Snodgrass, 1990), as was found with the present, originally blurred nature scenes.

- Experiment 4. Signal detection analyses showed a mean hit rate of $52 \%$ and a mean false alarm rate of $22 \%$. The d's were reliably above zero in each condition [Small Font/ Small Scene: $t(35)=6.75$, Small Font/Small Scene: $t(35)$ $=6.50$, Large Font $/$ Small Scene: $t(35)=5.59$, Large Font $/$ Large Scene: $t(35)=6.59$, all $p s<.001]$. No difference in $\mathrm{d}$ 's were observed based on font size or scene size, nor was there an interaction, $F_{\mathrm{S}}<1$. The $\mathrm{Cs}$ indicated a

\begin{tabular}{lll}
\hline adult & habit & rifle \\
badge & hood & romance \\
belly & host & rubber \\
benefit & hostage & sauce \\
bush & junk & seal \\
charm & knee & shark \\
costume & liver & singer \\
cotton & locker & skull \\
customer & noble & soap \\
deputy & option & status \\
dough & patrol & towel \\
exit & pitch & whistle \\
guilt & prayer & booth \\
guitar & product & clerk \\
\hline
\end{tabular}


conservative response bias in each condition [Small Font/ Small Scene: $t(35)=6.58$, Small Font/Small Scene: $t(35)$ $=5.42$, Large Font $/$ Small Scene: $t(35)=4.72$, Large Font $/$ Large Scene: $t(35)=4.97$, all $p s<.001]$. Cs did not change based on font size or scene size, nor was there an interaction, $F \mathrm{~s}<1$. Because differences in encoding and retrieval conditions did not favor one manipulation over the other in this experiment (e.g., Tulving \& Thomson, 1973), these results are consistent with our hypothesis in Experiment 2 that participants' d's decreased and they became more biased (Feenan \& Snodgrass, 1990) because of seeing formerly blurred backgrounds as clear ones at test (whereas clear backgrounds did not change at test).

- Experiment 5. Signal detection analyses showed a mean hit rate of $47 \%$ and mean false alarm rate of $35 \%$. The d's were reliably above zero in each condition [Small, Blurred Scene: $t(31)=1.92$; Small, Clear Scene: $t(31)=2.32$; Large, Blurred Scene: $t(31)=2.88$; Large, Clear Scene: $t(31)=7.15$, all $p s<.05]$. Large scenes were better recognized than small scenes, $F(1,31)=9.21, M S E=.219, p=$ $.005, \eta_{p}^{2}=.23$, and clear scenes were better recognized than blurred scenes, $F(1,31)=7.49, M S E=.260, p=$ $.010, \eta_{p}^{2}=.19$. A scene size by blur interaction indicated particularly high d' for large/clear scenes, $F(1,31)=4.92$, $M S E=.298, p=.034, \eta_{p}^{2}=.13$. We speculate that perhaps large and/or clear scenes better captured attention, however, why scene size failed to show even a small effect on JOLs or why a similar effect of scene size on d' was not found in Experiment 4 remains unclear with this explanation. The Cs indicated a conservative response bias in each condition [Small, Blurred Scene: $t(31)=2.43$; Small, Clear Scene: $t(31)=3.17$; Large, Blurred Scene: $t(31)=$ 2.11; Large, Clear Scene: $t(31)=3.77$, all $p s<.05$ ], but did not appear to respond differently as a function of condition (Scene Size: $F<1$, Scene Blur: $F(1,31)=2.23, M S E=$ $.145, p=.14$; Scene Size X Scene Blur: $F<1$ ).

\section{References}

Arbuckle, T. Y., \& Cuddy, L. L. (1969). Discrimination of item strength at time of presentation. Journal of Experimental Psychology, 81(1), 126-131. doi:https://doi.org/10.1037/h0027455

Benjamin, A. S., Bjork, R. A., \& Schwartz, B. L. (1998). The mismeasure of memory: when retrieval fluency is misleading as a metamnemonic index. Journal of Experimental Psychology: General, 127(1), 55-68. doi:https://doi.org/10.1037/0096-3445. 127.1.55

Besken, M. (2016). Picture-perfect is not perfect for metamemory: Testing the perceptual fluency hypothesis with degraded images. Journal of Experimental Psychology: Learning, Memory, and Cognition, 42(9), 1417-1433. doi:https://doi.org/10.1037/ xlm0000246
Besken, M., \& Mulligan, N. W. (2013). Easily perceived, easily remembered? Perceptual interference produces a double dissociation between metamemory and memory performance. Memory \& Cognition, 41(6), 897-903. doi:https://doi.org/10.3758/s13421013-0307-8

Brysbaert, M., \& New, B. (2009). Moving beyond Kučera and Francis: A critical evaluation of current word frequency norms and the introduction of a new and improved word frequency measure for American English. Behavior Research Methods, 41(4), 977-990. doi:https://doi.org/10.3758/BRM.41.4.977

Castel, A. D. (2008). Metacognition and learning about primacy and recency effects in free recall: The utilization of intrinsic and extrinsic cues when making judgments of learning. Memory \& Cognition, 36(2), 429-437. doi:https://doi.org/10.3758/MC.36.2.429

Cohen, J. (1988). Statistical power analysis for the behavioral sciences (2nd ed.). Hillsdale, NJ: Lawrence Erlbaum Associates.

Chalfonte, B.L., \& Johnson, M. K. (1996). Feature memory and binding in young and older adults. Memory \& Cognition, 24(4), 403-416. doi:https://doi.org/10.3758/BF03200930

Davenport, J. L., \& Potter, M. C. (2004). Scene consistency in object and background perception. Psychological Science, 15(8), 559-564. doi: https://doi.org/10.1111/j.0956-7976.2004.00719.x

Del Re, A. C. (2015). A practical tutorial on conducting meta-analysis in R. The Quantitative Methods for Psychology, 11(1), 37-50. doi: https://doi.org/10.20982/tqmp.11.1.p037

Dunlosky, J., Hunt, R. R., \& Clark, E. (2000). Is perceptual salience needed in explanations of the isolation effect? Journal of Experimental Psychology: Learning, Memory, and Cognition, 26(3), 649. doi:https://doi.org/10.1037/0278-7393.26.3.649

Dunlosky, J., \& Matvey, G. (2001). Empirical analysis of the intrinsicextrinsic distinction of judgments of learning (JOLs): Effects of relatedness and serial position on JOLs. Journal of Experimental Psychology: Learning, Memory, and Cognition, 27(5), 1180-1191. doi:https://doi.org/10.1037/0278-7393.27.5.1180

Dunlosky, J., \& Nelson, T. O. (1994). Does the sensitivity of judgments of learning (JOLs) to the effects of various study activities depend on when the JOLs occur? Journal of Memory and Language, 33(4), 545-565. doi:https://doi.org/10.1006/jmla.1994.1026

Faul, F., Erdfelder, E., Buchner, A., \& Lang, A. G. (2009). Statistical power analyses using $\mathrm{G}^{*}$ Power 3.1: Tests for correlation and regression analyses. Behavior Research Methods, 41(4), 1149-1160. doi:https://doi.org/10.3758/BRM.41.4.1149

Feenan, K., \& Snodgrass, J. G. (1990). The effect of context on discrimination and bias in recognition memory for pictures and words. Memory \& Cognition, 18(5), 515-527. doi:https://doi.org/10.3758/ BF03198484

Koriat, A. (1997). Monitoring one's own knowledge during study: A cueutilization approach to judgments of learning. Journal of Experimental Psychology: General, 126(4), 349-370. doi:https:// doi.org/10.1037/0096-3445.126.4.349

Koriat, A. (2008). Easy comes, easy goes? The link between learning and remembering and its exploitation in metacognition. Memory \& Cognition, 36(2), 416-428. doi:https://doi.org/10.3758/MC.36.2. 416

Lange, E. B. (2005). Disruption of attention by irrelevant stimuli in serial recall. Journal of Memory and Language, 53(4), 513-531. doi: https://doi.org/10.1016/j.jml.2005.07.002

Luna, K., Martín-Luengo, B., \& Albuquerque, P. B. (2017). Do delayed judgments of learning reduce metamemory illusions? A meta-analysis. The Quarterly Journal of Experimental Psychology, 71(7), 1626-1636. doi:https://doi.org/10.1080/17470218.2017.1343362

Magreehan, D. A., Serra, M. J., Schwartz, N. H., \& Narciss, S. (2016). Further boundary conditions for the effects of perceptual disfluency on judgments of learning. Metacognition and Learning, 11(1), 3556. doi:https://doi.org/10.1007/s11409-015-9147-1 
Matvey, G., Dunlosky, J., \& Schwartz, B. (2006). The effects of categorical relatedness on judgements of learning (JOLs). Memory, 14(2), 253-261. doi:https://doi.org/10.1080/09658210500216844

McCabe, J. (2011). Metacognitive awareness of learning strategies in undergraduates. Memory \& Cognition, 39(3), 462-476. doi:https:// doi.org/10.3758/s13421-010-0035-2

McGeoch, J.A. (1942). The psychology of human learning. Oxford, England: Longmans, Green.

Mueller, M.L. \& Dunlosky, J. (2017). How beliefs can impact judgments of learning: Evaluating analytic processing theory with beliefs about fluency. Journal of Memory and Language, 93, 245-258. doi: https://doi.org/10.1016/j.jml.2016.10.008.

Mueller, M. L., Dunlosky, J., Tauber, S. K., \& Rhodes, M. G. (2014). The font-size effect on judgments of learning: Does it exemplify fluency effects or reflect people's beliefs about memory? Journal of Memory and Language, 70, 1-12. doi:https://doi.org/10.1016/j.jml.2013.09. 007

Peynircioğlu, Z. F. \& Tatz, J. R. (2019). Intensifying the intensity illusion in judgments of learning: Modality and cue combinations. Memory \& Cognition, 47(3), 412-419. doi:https://doi.org/10.3758/s13421018-0875-8

Pieger, E., Mengelkamp, C., \& Bannert, M. (2016). Metacognitive judgments and disfluency - Does disfluency lead to more accurate judgments, better control, and better performance? Learning and Instruction, 44, 31-40. doi:https://doi.org/10.1016/j.learninstruc. 2016.01.012

Rosner, T. M., Davis, H., \& Milliken, B. (2015). Perceptual blurring and recognition memory: A desirable difficulty effect revealed. Acta Psychologica, 160, 11-22. doi:https://doi.org/10.1016/j.actpsy. 2015.06.006

Rhodes, M. G. (2016). Judgments of learning: Methods, data, and theory. In J. Dunlosky \& S.K. Tauber (Eds.), The Oxford handbook of metamemory (pp. 65-80). New York, NY: Oxford University Press.

Rhodes, M. G., \& Castel, A. D. (2008). Memory predictions are influenced by perceptual information: Evidence for metacognitive illusions. Journal of Experimental Psychology: General, 137(4), 615-625. doi:https://doi.org/10.1037/a0013684

Saenz, G. D., \& Smith, S. M. (2018). Testing Judgments of Learning in New Contexts to Reduce Confidence. Journal of Applied Research in Memory and Cognition, 7(4), 540-551. doi:https://doi.org/10. 1016/j.jarmac.2018.07.003

Sungkhasettee, V. W., Friedman, M. C., \& Castel, A. D. (2011). Memory and metamemory for inverted words: Illusions of competency and desirable difficulties. Psychonomic Bulletin \& Review, 18(5), 973. doi:https://doi.org/10.3758/s13423-011-0114-9

Susser, J. A., Mulligan, N. W., \& Besken, M. (2013). The effects of list composition and perceptual fluency on judgments of learning (JOLs). Memory \& Cognition, 41(7), 1000-1011. doi:https://doi. org/10.3758/s13421-013-0323-8

Tulving, E., \& Thomson, D. M. (1973). Encoding specificity and retrieval processes in episodic memory. Psychological Review, 80(5), 352373. doi:https://doi.org/10.1037/h0020071

Undorf, M., Söllner, A., \& Bröder, A. (2018). Simultaneous utilization of multiple cues in judgments of learning. Memory \& Cognition, 46(4), 507-519. doi:https://doi.org/10.3758/s13421-017-0780-6

Undorf, M., Zimdahl, M. F., \& Bernstein, D. M. (2017). Perceptual fluency contributes to effects of stimulus size on judgments of learning. Journal of Memory and Language, 92, 293-304. doi:https://doi.org/ 10.1016/j.jml.2016.07.003

Yue, C. L., Castel, A. D., \& Bjork, R. A. (2013). When disfluency isand is not - a desirable difficulty: The influence of typeface clarity on metacognitive judgments and memory. Memory \& Cognition, 41(2), 229-241. doi:https://doi.org/10.3758/s13421-012-0255-8

Zechmeister, E. B., \& Shaughnessy, J. J. (1980). When you know that you know and when you think that you know but you don't. Bulletin of the Psychonomic Society, 15, 41-44. doi:https://doi.org/10.3758/ BF03329756

Publisher's note Springer Nature remains neutral with regard to jurisdictional claims in published maps and institutional affiliations. 